\title{
A Novel Method for Measurement Gastric Emptying Using Three-dimensional Micro Computed Tomography in Small Live Animal
}

TO THE EDITOR: Alteration of gastric emptying $(\mathrm{GE})$ is one of important pathophysiology in functional gastrointestinal disorders such as functional dyspepsia and diabetic gastroparesis. ${ }^{1}$ Therefore, it is essential to measure GE in investigating the pathophysiology of disease or new drug efficacy in the translational animal experiment. In small animals such as mice or rat, phenol red recovery with photospectrometry from stomach has been used for liquid GE. ${ }^{2}$ For solid GE, measurement of remnant gastric food after spontaneous ingestion of normal rat chow has been used. ${ }^{3}$ However, these methods require the sacrifice of an experimental animal and obtain only one-time point GE value. Recently, it has been recommended to minimize the sacrifice of an animal in the basic experiment. The non-invasive ${ }^{14} \mathrm{C}$-octanoic acid breath test was recently applied to rats and it allows repetitive measurements of GE. ${ }^{4}$ However, it is not possible to measure gastric volume in a live animal.

We recently demonstrated that gastric volume could be measured in the rat using three-dimensional (3D) micro-CT (PolarisG90, Nano Focus Ray, Iksan, Korea; Fig. 1). ${ }^{5}$ Based on the experience of the previous experiment, we thought it would be possible to

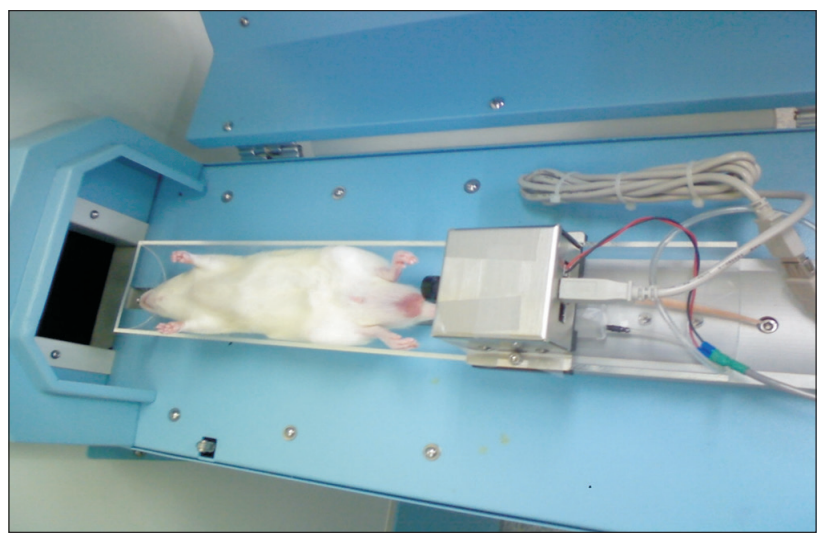

Figure 1. Scanning micro-CT in rat under anesthesia using PolarisG90. measure $\mathrm{GE}$ with $3 \mathrm{D}$ micro-CT in rat without sacrifice.

We performed a preliminary experiment on whether 3D microCT can measure GE change by drugs in Sprague-Dawley rat (210-310 g). Rats were divided into control, delayed GE (DGE, pre-treated with atropine), and enhanced GE (EGE, pre-treated with bethanechol) groups. After overnight fasting, distilled water, atropine $(7.5 \mathrm{mg} / \mathrm{kg})$, and bethanechol $(1 \mathrm{mg} / \mathrm{kg})$ was given by intraperitoneal injection in each group, respectively. After 20 minutes of injection, $3 \mathrm{~mL}$ of radiopaque semisolid food was fed into the stomach by gastric gavage under isoflurane anesthesia. Radiopaque semisolid food was a mixture of commercial food thickener containing carbohydrate (EG, 20 kcal/6 g; Goodseed company, Seoul, Korea) and radio-contrast dye (easySB, $30 \mathrm{~g}$ barium $/ 100 \mathrm{~mL}$; Taejoon pharm, Seoul, Korea). The calorie of final semi-solid food was 167 $\mathrm{cal} / \mathrm{mL}$. Immediately after gastric gavage, baseline CT (fed volume) was performed, and after 1 hour freely moving time, second CT (residual volume) was performed. Conventional CT image was converted to a 3D image, and the volume of radiopaque gastric food was measured (Fig. 2). GE rate was calculated as [(fed volume -
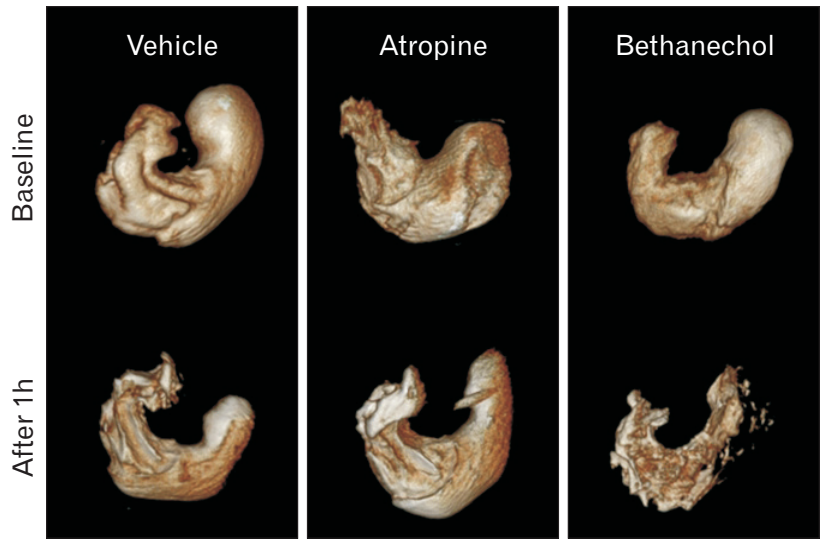

Figure 2. Representative 3-dimensional images of gastric volume change in 3 groups. 

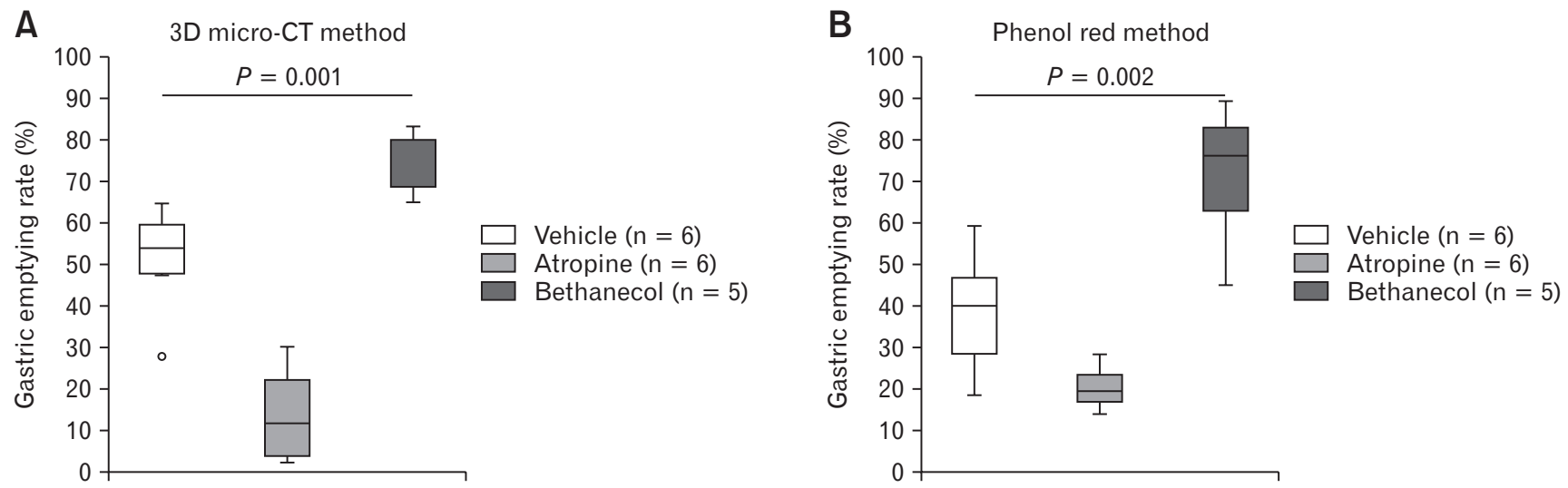

Figure 3. The gastric emptying (GE) rate in the rat. GE rated significantly decreased by atropine and increased by bethanechol. (A) GE rate measured by 3-dimensional micro-CT. (B) GE rate measured by phenol red photospectrometry. Data are expressed as the mean \pm range and statistical differences in the data were evaluated using the Kruskal-Wallis test.

residual volume $/$ fed volume $\times 100]$. Mean fed food volume on baseline CT was $3.00 \pm 0.02 \mathrm{~mL}, 2.99 \pm 0.05 \mathrm{~mL}$, and $2.41 \pm$ $0.71 \mathrm{~mL}$ and mean residual volume on second CT was $1.47 \pm$ $0.40 \mathrm{~mL}, 2.59 \pm 0.35 \mathrm{~mL}$, and $0.74 \pm 0.24 \mathrm{~mL}$ in control, DGE, and EGE groups, respectively. Because the emptying started very quickly and the fed volume was less than $3 \mathrm{~mL}$ in EGE group, the fed volume was unified to $3 \mathrm{~mL}$ in all groups when calculating the $\mathrm{GE}$ rate. The mean GE rate at 1 hour calculated using the volume of $3 \mathrm{D}$ micro-CT image was $51.1 \pm 13.2 \%, 13.8 \pm 11.6 \%$, and $75.3 \pm 8.1 \%$ in control, DGE, and EGE group, respectively. The difference was statistically significant (Fig. 3A). We performed the same experiment using the traditional phenol red method and observed a similar pattern (Fig. 3B).

Micro CT for the small animal has been used over 30 years in translational research; however, the usage of this technique limited to the anatomical change such as bone, vascular structure, and solid organ. ${ }^{6,7}$ We applied 3D micro-CT to gastric functional measurement and demonstrated that the GE rate could be calculated by CT data in the rat. Moreover, the GE pattern by drugs in 3D microCT experiment was comparable with those in the experiment with phenol red method. The limitation of this method is that it requires anesthesia, which can affect gastrointestinal motility.

In conclusion, the novel method using 3D micro-CT can measure GE serially in the same animal without sacrifice. Therefore it could be used to study the pathogenesis of functional gastric disorders and to investigate the efficacy of a new drug with decreasing animal sacrifice as well as repetitive measurement.

Yong Sung Kim, ${ }^{1}$ Seong Hoon Park, ${ }_{1}^{2}$ Eul-sig Choi, ${ }^{3}$ and Suck Chei Choi ${ }^{1 *}$
${ }^{1}$ Department of Gastroenterology and Digestive Disease Research Institute, Iksan, Jeonlabuk-do, Korea; ${ }^{2}$ Department of Radiology and Institute for Metabolic Disease, Iksan, Jeonlabuk-do, Korea; and ${ }^{3}$ Brain Science Institute, School of Medicine, Wonkwang University, Iksan, Jeonlabuk-do, Korea

1. Tack J. Gastric motor and sensory function. Curr Opin Gastroenterol 2009;25:557-565.

2. Czimmer J, Million M, Taché Y. Urocortin 2 acts centrally to delay gastric emptying through sympathetic pathways while $\mathrm{CRF}$ and urocortin 1 inhibitory actions are vagal dependent in rats. Am J Physiol Gastrointest Liver Physiol 2006;290:G511-G518.

3. Seto K, Sasaki T, Katsunuma K, Kobayashi N, Tanaka K, Tack J. Acotiamide hydrochloride (Z-338), a novel prokinetic agent, restores delayed gastric emptying and feeding inhibition induced by restraint stress in rats. Neurogastroenterol Motil 2008;20:1051-1059.

4. Demedts I, Vanormelingen C, Van Billoen H, et al. Validation of octanoate breath test for measuring gastric emptying in rats. J Neurogastroenterol Motil 2013;19:171-178.

5. Kim YS, Lee MY, Park JS, et al. Effect of DA-9701 on feeding inhibition induced by acute restraint stress in rats. Korean J Helicobacter Up Gastrointest Res 2018;18:50-55.

6. Schambach SJ, Bag S, Schilling L, Groden C, Brockmann MA. Application of micro-CT in small animal imaging. Methods 2010;50:2-13

7. Kim HW, Cai QY, Jun HY, et al. Micro-CT imaging with a hepatocyteselective contrast agent for detecting liver metastasis in living mice. Acad Radiol 2008;15:1282-1290.

Financial support: None: This work supported by Wonkwang University 2018 (C.S.C.).

Conflicts of interest: None.

Author contributions: Yong Sung Kim: study design, data analysis, and drafting and finalizing manuscript; Seong Hoon Park and Eulsig Choi: perform an experiment, data collection, data analysis, and drafting the manuscript; and Suck Chei Choi: study design and finalizing the manuscript 